\title{
Advanced Positive Airway Pressure Modalities
}

\author{
Gregory B. Bierer ${ }^{1} \cdot$ Armand Ryden $^{2} \cdot$ Ravi S. Aysola ${ }^{3}$
}

Published online: 31 October 2015

(C) Springer International Publishing AG 2015

\begin{abstract}
Auto-adjusting positive airway pressure (PAP) devices are increasingly becoming the first line treatment for obstructive sleep apnea (OSA). More complex sleepdisordered breathing such as Cheyne-Stokes respirations and central sleep apnea related to heart failure as well as complex sleep-disordered breathing related to chronic hypoventilation syndromes may require the use of advanced positive airway pressure modalities such as automated servo ventilation (ASV) and volume targeted pressure limited ventilation. Identifying patients with complex sleep-disordered breathing that will benefit from treatment with advanced modalities remains an area of active investigation. A recent and surprising
\end{abstract}

This article is part of the Topical Collection on Sleep Related Breathing Disorders

Ravi S. Aysola

raysola@mednet.ucla.edu

Gregory B. Bierer

gbierer@mednet.ucla.edu

Armand Ryden

Division of Pulmonary and Critical Care Medicine, Clinical Immunology and Allergy, UCLA Pulmonary Sleep Medicine Program, David Geffen School of Medicine at UCLA, 10833 Le Conte Ave, Room 37-131 CHS, Los Angeles, CA 90095-1690, USA

2 Division of Pulmonary, Critical Care \& Sleep Medicine, West Los Angeles VA Medical Center, The David Geffen School of Medicine at UCLA, 11301 Wilshire Blvd (111Q), Building 500, Room 3013B, Los Angeles, CA 90073, USA

3 Division of Pulmonary and Critical Care Medicine, Clinical Immunology and Allergy, UCLA Pulmonary Sleep Medicine Program, UCLA Santa Monica Pulmonary \& Sleep Medicine Clinic, David Geffen School of Medicine, 10833 Le Conte Ave, Room 37-131 CHS, Los Angeles, CA 90095-1690, USA development found ASV to be associated with an increased risk of sudden cardiac death when used to treat central sleep apnea in patients with symptomatic systolic heart failure and an ejection fraction less than $45 \%$. The optimal treatment of central sleep apnea in heart failure and the role of ASV are currently in question. The following chapter is intended to provide the clinician with a description of newer PAP modalities; a review of evidence supported indications for use as well as provide a framework for managing patients with advanced positive airway pressure therapy.

Keywords Positive airway pressure therapy - Auto adjusting • Servo ventilation $\cdot$ Sleep-disordered breathing

\begin{tabular}{ll}
\multicolumn{2}{l}{ Abbreviations } \\
OSA & Obstructive sleep apnea \\
PAP & Positive airway pressure \\
SV & Servo ventilation \\
APAP & Auto-adjusting positive airway pressure \\
CompSAS & Complex sleep apnea syndrome
\end{tabular}

\section{Introduction}

Since its introduction by Colin Sullivan in 1981, continuous positive airway pressure (CPAP) has been the standard therapy for obstructive sleep apnea $[1,2]$. Newer devices equipped with advanced microprocessors and capable of more sensitive airflow detection are able to modulate the pressure that is delivered in more sophisticated and subtle ways. The most commonly used advanced modality is auto-adjusting positive airway pressure (APAP). Advanced flow-based servo and volume targeted pressure support modes have been developed to modulate or augment ventilation to treat more complex sleep- 
disordered breathing. We will discuss the technical aspects of these devices and evidence for their use in sleep-disordered breathing syndromes.

\section{Auto-Adjusting PAP}

\section{General Principles}

Home sleep apnea testing and subsequent treatment with autoadjusting positive airway pressure (APAP) is becoming the standard pathway for treatment of obstructive sleep apnea. Though appealing to payers and providers, not all patients are appropriate for APAP treatment. APAP is not recommended for patients with comorbid congestive heart failure (CHF), intrinsic lung disease, and hypoventilation as stated in the American Academy of Sleep Medicine (AASM) practice parameters from 2008 [3], though subsequent research has shown that APAP along with home sleep testing may be non-inferior to polysomnography (PSG) titration in unselected patient populations [4]. Further research is needed to determine if home sleep apnea testing and treatment with APAP in selected patients with cardiopulmonary comorbidities (CHF and COPD) is safe and effective. A major concern is the ability of newer generation home sleep apnea tests and APAP devices to detect and accurately characterize respiratory events (e.g., varying degrees of obstructive versus central).

APAP devices analyze the flow signal to adjust pressure to an optimal setting at any given point in time. Determination of the optimal setting is based on proprietary algorithms unique to the specific device manufacturer. The Respironics REMstar Auto device, for example, employs a proactive algorithm based on the contour of the flow signal [5]. The device increases the pressure by $1.5 \mathrm{~cm} \mathrm{H}_{2} \mathrm{O}$ over 3 min to probe for an improvement in the airflow signal that determines whether the device stays at the higher pressure or reverts to the starting pressure. Conversely, the device also decreases the pressure until airflow limitation is detected [5]. If an obstructive respiratory event is detected, the device reacts by increasing the pressure within the search algorithm. Central apneas (CA) are detected by flow response to test pulses of pressure and are characterized as "clear airway apnea," to which the device is not to generate a pressure increase [5]. The detection and response algorithms are patented and vary among manufacturers. The ResMed AutoSet uses forced oscillation of small pulses at $4 \mathrm{~Hz}$ rather than larger discrete test pulses to detect an open airway [6]. ResMed AutoSet also responds to flow limitation and apneas but on a breath by breath basis, rather than in an active search [6]. These two devices are presented as examples because they are the most commonly used in the USA. The key point is that APAP devices from different manufacturers may perform differently.
Bench studies evaluating the response of different APAP devices to a range of breathing patterns have shown that the responses can be quite variable [7]. A recent bench investigation of eleven APAP devices showed that only five of the devices improved airflow to at least $70 \%$ of baseline breathing amplitude. The majority of devices did not reduce AHI to $<5 / \mathrm{h}$, and three devices underestimated AHI by $>10 \%$. Five devices increased pressure during snoring and seven devices increased pressure during central apneas. Only five of the APAP machines studied achieved treatment efficacy of $>90 \%$, and seven devices accurately identified AHI $>90 \%$ of the time [8]. These studies illustrate the variance between devices in event detection and response algorithm. Of course, the PAP-derived estimates of AHI are fundamentally different from the AHI observed during polysomnography (PSG) in that they only rely on airflow and use a proprietary algorithm [9]. Studies have shown close correlations between APAPestimated and PSG-derived AHI values for specific devices in patients without central apnea at baseline or treatmentemergent central apnea [10]. Other studies comparing APAP-detected AHI to PSG-scored AHI during attended PSG have shown that APAP-detected AHI overestimates but correlates closely with PSG AHI $[9,11]$. The overestimation of AHI has been found to be greatest at low values [12]. There have been conflicting findings regarding the sensitivity and specificity of device AHI for determining residual elevated AHI. One ROC analysis showed than an APAP AHI of six event/h had excellent sensitivity and specificity $(0.92$ and 0.90 ) to detect a PSG AHI $>5$. An analysis of a multicenter Respironics trial involving 115 patients found that a device AHI $>10$ had a 0.58 sensitivity and 0.94 specificity for PSG AHI $>10$ [13]. A retrospective review of 176 unselected patients comparing simultaneous APAP (ResMed) detected AHI and PSG AHI showed that only $58 \%$ had an adequate titration with APAP. A history of cardiac disease, baseline elevated central apnea index, and arousal index were predictors of APAP titration failure [14]. Similarly, Donotti et al. also found that APAP can fail to detect and respond to events in some patients leading to misclassification regarding residual AHI [15].

Air leak is an important factor that can potentially limit the APAP device's ability to detect and appropriately respond to respiratory events. A bench study found that older generation devices that used pressure-based algorithms responded to artificial snoring, but failed to respond to other flow-limiting respiratory events [16]. Newer generation devices that use flow detection algorithms were shown to accurately respond to obstructive respiratory events until leak rates exceeded $30 \mathrm{~L} / \mathrm{min}$ and that high leak resulted in an overestimate of the delivered pressure [16] Leak is associated with lower APAP-delivered pressure, higher residual AHI, and worse adherence [17]. It is important to remember that expected leak is also a function of pressure and the mask interface used. 


\section{APAP as Treatment for OSA}

APAP is an alternative to conventional CPAP for long-term therapy for obstructive sleep apnea. Potential advantages include improved comfort and adherence by minimizing unnecessarily high pressure. Most studies have shown only small differences in adherence between fixed CPAP and APAP. Early, small studies suggested that APAP may have advantages over CPAP in terms of adherence $[18,19]$. A more recent randomized 6-week trial in almost 200 unselected patients showed a more modest difference in adherence of $0.2 \mathrm{~h}$ per night $(12 \mathrm{~min}$ ) and a small difference in sleepiness between APAP and CPAP [20]. Meta-analyses of randomized trials in mostly CPAP naive patients have consistently shown small but usually statistically significant differences in adherence of 11-14 min per night favoring APAP [21-24]. A Cochrane review found no difference in residual AHI; however, there was a very small but significant difference in the Epworth Sleepiness Scale of 0.5 points favoring APAP [22].

The efficacy of APAP in treating obstructive sleep apnea (OSA) is critical for its acceptance as a therapy. A small study in morbidly obese patients $(n=12)$ requiring high therapeutic pressures $\left(>14 \mathrm{~cm} \mathrm{H}_{2} \mathrm{O}\right)$, showed that APAP (ResMed) was equally effective to fixed CPAP at reducing AHI, and did so at a lower average pressure [25]. A recent randomized controlled trial of Respironics A-flex (APAP with flexible expiratory pressure) versus CPAP showed an advantage of CPAP in regards to residual $\mathrm{AHI}$ and mean oxygen saturation at the start of the trial, but this advantage dissipated at 180 days [26]. Scores of sleepiness, results of psychomotor vigilance testing and blood pressure were no different between the groups after 180 days [26]. In this relatively unselected group, efficacy was essentially the same. An earlier study of patients with high within-night pressure variability based on APAP titration were randomized to fixed CPAP or APAP [27]. In this group of patients, sleepiness scores were better on APAP than on CPAP. In a trial of patients requiring higher pressures, APAP resulted in a greater than 30 min per night improvement in adherence and lower average pressure than with fixed CPAP, with better self-reported sleep quality scores $[18,20]$. These findings suggest that while there may be minimal differences between CPAP and APAP for unselected patients, there may be groups of patients who may do better on APAP, including those with significant pressure variability, or those who require high therapeutic pressures. A recent investigation of 156 patients diagnosed with OSA by home sleep testing showed equivalence of APAP therapy compared to CPAP after an attended titration study [28].

A relatively few, small studies have shown mixed results in regards to the effects of APAP versus CPAP on the cardiovascular and metabolic consequences of obstructive sleep apnea. One study looking at CPAP versus APAP in 40 patients showed that CPAP resulted in reductions in blood pressure and a measure of insulin resistance (HOMA-IR) while APAP did not [29]. A follow-up study by Patruno et al. suggests that APAP results in greater sympathetic activation and lower cardiorespiratory coupling than CPAP [30]. These studies were in small patient populations and only applicable to one device (ResMed). Larger studies are needed to determine if APAP is as effective as CPAP in reducing health risks and improving health outcomes in patients with OSA.

As previously discussed, APAP devices use proprietary algorithms to adjust pressure and not all devices are equal in regards to patient adherence, comfort, and residual respiratory events [31,32]. There have been few large studies comparing devices from different manufactures with regards to these outcomes. A head to head comparison of ResMed and Respironics APAP devices in a randomized crossover trial showed that the Respironics device achieved a lower AHI with a lower average pressure, but there were no significant differences in sleep efficiency or subjective ratings of these devices [33]. The authors speculated that the higher pressures of the ResMed device could be associated with failure to drop pressure in response to artifact or leak, which could then contribute to higher leak and reflex pharyngeal constriction. Additionally, device technology is constantly evolving, which may render previous studies obsolete.

\section{APAP as an Alternative to CPAP Titration}

APAP may be an attractive alternative to traditional CPAP titration. The American Academy of Sleep Medicine (AASM) practice parameters state that APAP may be used in attended polysomnogram titration, while it is an option (weaker recommendation) that certain APAP devices may be used at home in unattended PAP titration in moderate to severe OSA [3]. A small study of 20 patients showed APAP to be as effective as manual CPAP titration for AHI reduction, adherence, and clinical efficacy with a trend towards increased sleep the first titration night favoring APAP [34]. Mulgrew et al. performed a larger trial comparing APAP titration versus CPAP as part of an algorithm investigating in-lab versus out of center testing [35]. At 3 months, there was no difference in AHI, quality of life, or sleepiness, but adherence was better in the out of center arm [35]. A more recent meta-analysis of manual CPAP titration versus auto-titration found that they were equally effective for residual AHI, reduction in sleepiness, patient acceptance, and adherence [36]. There was significant heterogeneity in this meta-analysis, possibly due to the range of devices tested. A recent study using a single night titration between ResMed and Respironics devices showed that the fixed pressure recommended was very similar for both devices and resulted in an AHI $<5 / \mathrm{h}$ [37]. Generally, when APAP is used as an alternative to CPAP titration, the 90 or $95 \%$ percentile 
pressure (i.e., the pressure required to eliminate 90 or $95 \%$ of respiratory events) is used to determine the fixed CPAP setting. Little is known regarding how many nights the APAP device should be worn to determine this pressure.

\section{General Recommendations for Prescribing APAP}

Most of the studies of APAP have used fairly large ranges of pressure. However, there may be advantages to prescribing a more narrow pressure range. In some patients, starting at a setting that is too low may disturb sleep by allowing respiratory events while the pressure is at a sub-therapeutic level. It is also theoretically possible that failure of the device algorithms may lead to over-titration resulting in excessive pressure, leak, and patient discomfort.

Current devices provide detailed data regarding adherence and efficacy. AHI (including central apnea and periodic breathing), leak, sensed snoring, as well as the 90th or 95th percentile pressure are typically reported. Clinicians should take into account the estimate of leak, duration of use, and actual sleep time when making treatment decisions based on data card reports. The device algorithms for pressure titration are less accurate in the presence of significant leaks. Devices that incorporate cloud-based reporting and management systems are being utilized to develop telemedicine programs [38].

\section{Adaptive Servo Ventilation}

\section{General Principles}

Adaptive servo ventilation (ASV) is a type of bilevel device that provides variable pressure support ventilation in response to the patient's breathing. Most current devices utilize APAP algorithms generate an expiratory positive airway pressure (EPAP) at a level that eliminates obstructive events and flow limitation. Pressure support is increased in response to hypopnea and decreased in response to hyperpnea, in an effort to stabilize fluctuating ventilation. A back-up rate (either prescribed by the provider or automatically generated by the machine) will ventilate the patient during central apneas. ASV is generally not appropriate for chronic hypoventilation syndromes as device-targeted minute ventilation is less than the previous minute ventilation.

Devices may differ in their method of detecting ventilation, respiratory events and response algorithms [39]. The clinical consequences of the variation is unclear.

\section{ASV for Cheyne-Stokes Respiration}

There has been great interest in applying ASV to treat central sleep apnea and Cheyne-Stokes respiration in patients with heart failure. Small, early studies showed that ASV was more effective than oxygen, CPAP, and bi-level positive airway pressure (BPAP) at reducing central AHI [39, 40]. Subsequent studies showed that ASV improved left ventricular ejection fraction and 6-min walk distance in patients with heart failure [41-43] A recent meta-analysis of studies comparing ASV to non-ASV therapy including CPAP, $\mathrm{O}_{2}$, BPAP, and no treatment showed that ASV more effectively reduced AHI [44]. Similarly, ASV has been shown to be beneficial in heart failure with preserved ejection fraction (LVEF $>50 \%$ ) [45]. ASV has also been linked to improvement in cardiorenal dysfunction in patients with $\mathrm{CHF}$ and chronic kidney disease [46].

The most prominent trial looking at PAP therapy for CHFrelated central sleep apnea was the CANPAP trial. This multicenter trial randomized 258 patients to fixed CPAP or usual care and found that on average, fixed CPAP (not titrated) reduced AHI by $50 \%$ to around $20 / \mathrm{h}$, but did not result in a mortality benefit. Modest improvements in nocturnal oxygenation, ejection fraction, and 6-min walk distance were noted [47]. A secondary analysis of the trial showed that patients whose AHI was suppressed to $<15 / \mathrm{h}$ experienced an improvement in transplant-free survival compared to patients whose AHI remained $>15 / h$ on CPAP [48]. Since ASV has demonstrated superiority in suppressing Cheyne-Stokes respiration, there is interest in determining if a mortality benefit can be achieved with ASV. The SERVE-HF, sponsored by ResMed, is a large randomized control trial in patients with symptomatic heart failure and an ejection fraction $<45 \%$ assessing the impact of ASV therapy versus medical management for CSA/ CSR on heart transplant-free survival [49]. The preliminary data elicited a press release in May 2015 disclosing a $2.5 \%$ increase in absolute risk of cardiovascular death in the ASV group (10 versus $7.5 \%$ in control group). Publication of the study will allow for a greater understanding of the specific findings, but an immediate discontinuation of ASV in patients with an EF $<45 \%$ is recommended by the ResMed [50]. The FDA "strongly recommend clinicians adhere to the recommendations cautioning against the use of ASV therapy in patients with symptomatic chronic heart failure (NYHA 2-4) and reduced $\mathrm{LVEF} \leq 45 \%$, AND moderate to severe predominant central sleep apnea." [51] It is important to note that the ASV algorithm utilized in the SERVE-HF trial may not be identical to current software or future updates of the ResMed device. Respironics is currently enrolling patients in the ADVENT-HF trial with a similar design to the SERVE-HF trial [52].

\section{ASV for Treatment-Emergent Central Sleep Apnea}

Management of treatment-emergent central sleep apnea, also known as complex sleep apnea syndrome (CompSAS), is controversial [53]. The majority of such cases abate after treatment with fixed CPAP [54]. Although originally developed to 
treat Cheyne-Stokes respiration, ASV has been promoted as a therapy for persistent treatment-emergent central sleep apnea. An early study from the Mayo Clinic including a mix of patients, the majority of whom had CompSAS, showed that ASV significantly improved AHI and REM sleep compared to CPAP [55]. A small early study which assessed ASV for both Cheyne-Stokes respiration and treatment-emergent central apnea showed that ASV was superior to titrated BPAP in both groups [56]. Another small retrospective case series of patients titrated to ASV for treatment-emergent central sleep apnea that did not get better with CPAP therapy found that ASV could achieve an AHI $<5 / \mathrm{h}$ in $80 \%$ of patients and less than $10 / \mathrm{h}$ in $92 \%$, with the average AHI going from $44.4 / \mathrm{h}$ on standard PAP to $3.6 / \mathrm{h}$ on ASV [57]. Bi-level positive airway pressure (BPAP) can be considered to treat patients with CompSAS; however, there has been some association with worsening central apneas [58]. A randomized controlled trial comparing BPAP and ASV showed that after 6 weeks, ASV was clearly superior with regards to residual AHI and elimination of central events [59]. Currently available evidence suggests that ASV is effective at treating treatment-emergent central sleep apnea. However, given the transient nature of this condition, the ongoing need for ASV should be assessed after therapy initiation.

\section{ASV for Idiopathic and Opioid-Induced Central Sleep Apnea}

There is less evidence regarding the use of ASV for central sleep apnea induced by opiate use. Early reports from small studies showed conflicting results regarding the efficacy of ASV in treating opioid-induced central sleep apnea [60, 61]. A larger study compared ASV for central sleep apnea in 47 opioid patients and $61 \mathrm{CHF}$ patients who failed CPAP. An AHI less than 10 was achieved with ASV in approximately $60 \%$ of the opioid patients and $70 \%$ in the CHF patients [62]. Interestingly, lower BMI, higher serum bicarbonate values, and absence of Cheyne-Stokes respiration were predictors of successful ASV titration [62].

Similar to opioid-related central sleep apnea, there have been few reports of response to ASV in idiopathic central sleep apnea. One of the earliest case series showed an excellent response to ASV for idiopathic Cheyne-Stokes respiration in three patients [63]. A recent retrospective review of patients with idiopathic and opioid-induced central sleep apnea found that ASV resulted in an AHI $<10 / \mathrm{h}$ in $60 \%$ of opiate-induced CSA and $90 \%$ of idiopathic CSA [64]. However, two thirds of the patients in this study were effectively managed with standard CPAP or BPAP [64]. Another retrospective study compared central sleep apnea in patients with CHF versus nonCHF patients [65]. This study showed that ASV was highly efficacious in eliminating events for both groups of patients [65]. These findings suggest that ASV may apply to other populations with central sleep-disordered breathing that is not due to chronic respiratory failure.

\section{General Recommendations for Prescribing ASV}

ASV may be considered for patients with an EF $>45 \%$ and whose sleep-disordered breathing is not effectively controlled with CPAP, BPAP, or PAP with supplemental oxygen. The cost differential is significant between fixed CPAP and ASV. In the USA, CMS requires the following criteria be met to qualify for ASV therapy: in-laboratory polysomnography demonstrating a central and overall $\mathrm{AHI}>5$, with central AHI accounting for $>50 \%$ of the total AHI; presence of symptoms of excessive sleepiness or disturbed sleep; and demonstration that ASV significantly improved the sleep-disordered breathing. For treatment-emergent CSA, these polysomnographic conditions apply while on CPAP therapy for OSA.

Although ASV devices are automated, it is recommended that patients undergo in-laboratory titration to confirm efficacy. The titration is also important to assess for potential problems, including insufficient EPAP and mask leaks, as well as to demonstrate benefit of the therapy. Manufacturers of ASV devices have incorporated APAP algorithms to modify the EPAP for some devices [66]. Mask leaks tend to make ASV therapy problematic because the complex calculation and delivery of ventilation will be inaccurate. Therefore, addressing and troubleshooting mask leaks are of paramount importance during APAP titration.

\section{Bi-Level Positive Airway Pressure and Auto-Adjusting BPAP}

\section{Fixed Bi-Level Devices}

Bi-level positive airway pressure (BPAP) is a mode that alternates pressure settings on inspiration (IPAP) and expiration (EPAP), with the purported advantage of improving patient comfort and tolerance. Auto-adjusting BPAP modes have also been developed to further this goal. Though multiple manufacturers offer BPAP devices, there has not been convincing evidence that BPAP improves adherence or outcomes when compared to CPAP [22,67].

\section{AVAPS}

\section{General Principles}

Average volume assured pressure support (AVAPS) is an automatically titrating mode of BPAP, which automatically adjusts pressure support to target a set volume. The mode was developed to account for the changes in respiratory muscle 
load and respiratory drive that vary during sleep and may change during the course of disease.

Most studies comparing AVAPS to BPAP have shown modest improvements in nocturnal ventilation in those treated with AVAPS, without significant improvements in sleep quality or quality of life [68-71]. One study showed that patients with COPD and hypercapnia experienced better sleep efficiency with AVAPS versus BPAP [72].

AVAPS is most commonly used to treat chronic respiratory failure secondary to obesity hypoventilation in which CPAP is often inadequate to eliminate nocturnal oxygen desaturations [73]. Patients with chronic respiratory failure due to OHS, neuromuscular disease, and COPD may benefit from AVAPS therapy, though further studies are needed to determine whether AVAPS is genuinely superior to fixed BPAP. As with other algorithms, the AVAPS event detection algorithms are limited by mask leak and this should be assessed to avoid inappropriate changes in therapy.

\section{Conclusion}

Advanced positive airway pressure modalities are increasingly being used to expedite the diagnosis and treatment of OSA, as well as treat more complex sleep-related breathing disorders. Auto-PAP (APAP) is being employed by providers to tailor pressure settings, improve adherence, and to improve access to therapy when attended sleep studies are in short supply. As financial pressures push to move the diagnosis of OSA out of the sleep laboratory and into the home, APAP may have a growing role in titration and therapy.

Adaptive servo ventilation may yet find a role in improving complex sleep-disordered breathing, but with the current data from SERVE-HF the use of ASV in patients with an ejection fraction $<45 \%$ is not recommended. Further clarification and direction from the publication of key studies is eagerly awaited. Advanced ventilator support algorithms have the potential to enable more effective treatment of sleep-related hypoventilation syndromes in patients with morbid obesity, restrictive thoracic disease, and COPD. However, more evidence is required to see if they are indeed more promising than conventional bi-level positive airway pressure devices.

\section{Compliance with Ethical Standards}

Conflict of Interest Gregory B. Bierer, Armand Ryden, and Ravi S. Aysola declare that they do not have any conflicts of interest.

Human and Animal Rights and Informed Consent This article does not contain any studies with human or animal subjects performed by any of the authors.

\section{References}

1. Sullivan CE, Issa FG, Berthon-Jones M, Eves L. Reversal of obstructive sleep apnoea by continuous positive airway pressure applied through the nares. Lancet. 1981;1:862-5.

2. Epstein LJ, Kristo D, Strollo Jr PJ, et al. Clinical guideline for the evaluation, management and long-term care of obstructive sleep apnea in adults. J Clin Sleep Med. 2009;5:263-76.

3. Morgenthaler TI, Aurora RN, Brown T, et al. Practice parameters for the use of autotitrating continuous positive airway pressure devices for titrating pressures and treating adult patients with obstructive sleep apnea syndrome: an update for 2007. An American Academy of Sleep Medicine report. Sleep. 2008;31:141-7.

4. Kuna ST, Gurubhagavatula I, Maislin G, et al. Noninferiority of functional outcome in ambulatory management of obstructive sleep apnea. Am J Respir Crit Care Med. 2011;183:1238-44.

5. Respironics. Clinical applications guide REMstar Auto. 2009.

6. ResMed. S9 Series AutoSet Elite H5i Clinical Guide. 2010.

7. Farre R, Montserrat JM, Rigau J, Trepat X, Pinto P, Navajas D. Response of automatic continuous positive airway pressure devices to different sleep breathing patterns: a bench study. Am J Respir Crit Care Med. 2002;166:469-73.

8. Zhu K, Roisman G, Aouf S, Escourrou P. All APAPs are not equivalent for the treatment of sleep disordered breathing: a bench evaluation of eleven commercially available devices. J Clin Sleep Med. 2015;11(7):725-34.

9. Stepnowsky C, Zamora T, Barker R, Liu L, Sarmiento K. Accuracy of positive airway pressure device-measured apneas and hypopneas: role in treatment follow up. Sleep Disord. 2013;2013: 314589.

10. Ikeda Y, Kasai T, Kawana F, et al. Comparison between the apnea-hypopnea indices determined by the REMstar Auto M Series and those determined by standard in-laboratory polysomnography in patients with obstructive sleep apnea. Intern Med. 2012;51:2877-85.

11. Woodson BT, Saurejan A, Brusky LT, Han JK. Nonattended home automated continuous positive airway pressure titration: comparison with polysomnography. Otolaryngol Head Neck Surg. 2003;128:353-7.

12. Desai H, Patel A, Patel P, Grant BJ, Mador MJ. Accuracy of autotitrating CPAP to estimate the residual Apnea-Hypopnea Index in patients with obstructive sleep apnea on treatment with autotitrating CPAP. Sleep Breath. 2009;13:383-90.

13. Berry RB, Kushida CA, Kryger MH, Soto-Calderon H, Staley B, Kuna ST. Respiratory event detection by a positive airway pressure device. Sleep. 2012;35:361-7.

14. Huang HC, Hillman DR, McArdle N. Control of OSA during automatic positive airway pressure titration in a clinical case series: predictors and accuracy of device download data. Sleep. 2012;35: 1277-83A.

15. Denotti AL, Wong KK, Dungan 2nd GC, Gilholme JW, Marshall NS, Grunstein RR. Residual sleep-disordered breathing during autotitrating continuous positive airway pressure therapy. Eur Respir J. 2012;39:1391-7.

16. Coller D, Stanley D, Parthasarathy S. Effect of air leak on the performance of auto-PAP devices: a bench study. Sleep Breath. 2005;9:167-75.

17. Valentin A, Subramanian S, Quan SF, Berry RB, Parthasarathy S. Air leak is associated with poor adherence to autoPAP therapy. Sleep. 2011;34:801-6.

18. Massie CA, McArdle N, Hart RW, et al. Comparison between automatic and fixed positive airway pressure therapy in the home. Am J Respir Crit Care Med. 2003;167:20-3. 
19. Hukins C. Comparative study of autotitrating and fixed-pressure CPAP in the home: a randomized, single-blind crossover trial. Sleep. 2004;27:1512-7.

20. Vennelle M, White S, Riha RL, Mackay TW, Engleman HM, Douglas NJ. Randomized controlled trial of variable-pressure versus fixed-pressure continuous positive airway pressure (CPAP) treatment for patients with obstructive sleep apnea/hypopnea syndrome (OSAHS). Sleep. 2010;33:267-71.

21. Ip S, D'Ambrosio C, Patel K, et al. Auto-titrating versus fixed continuous positive airway pressure for the treatment of obstructive sleep apnea: a systematic review with meta-analyses. Syst Rev. 2012;1:20.

22. Smith I, Lasserson TJ. Pressure modification for improving usage of continuous positive airway pressure machines in adults with obstructive sleep apnoea. Cochrane Database Syst Rev. 2009: CD003531.

23. Xu T, Li T, Wei D, et al. Effect of automatic versus fixed continuous positive airway pressure for the treatment of obstructive sleep apnea: an up-to-date meta-analysis. Sleep Breath. 2012;16:1017-26.

24. Ayas NT, Patel SR, Malhotra A, et al. Auto-titrating versus standard continuous positive airway pressure for the treatment of obstructive sleep apnea: results of a meta-analysis. Sleep. 2004;27:249-53.

25. Bakker J, Campbell A, Neill A. Randomised controlled trial of auto-adjusting positive airway pressure in morbidly obese patients requiring high therapeutic pressure delivery. J Sleep Res. 2011;20: 233-40.

26. Kushida CA, Berry RB, Blau A, et al. Positive airway pressure initiation: a randomized controlled trial to assess the impact of therapy mode and titration process on efficacy, adherence, and outcomes. Sleep. 2011;34:1083-92.

27. Noseda A, Kempenaers C, Kerkhofs M, Braun S, Linkowski P, Jann E. Constant vs auto-continuous positive airway pressure in patients with sleep apnea hypopnea syndrome and a high variability in pressure requirement. Chest. 2004;126:31-7.

28. Berry RB, Sriram P. Auto adjusting positive airway pressure treatment for sleep apnea diagnosed by home sleep testing. J Clin Sleep Med. 2014;10(12):1269-75.

29. Patruno V, Aiolfi S, Costantino G, et al. Fixed and autoadjusting continuous positive airway pressure treatments are not similar in reducing cardiovascular risk factors in patients with obstructive sleep apnea. Chest. 2007;131:1393-9.

30. Patruno V, Tobaldini E, Bianchi AM, et al. Acute effects of autoadjusting and fixed continuous positive airway pressure treatments on cardiorespiratory coupling in obese patients with obstructive sleep apnea. Eur J Intern Med. 2014;25:164-8.

31. Nolan GM, Ryan S, O'Connor TM, McNicholas WT. Comparison of three auto-adjusting positive pressure devices in patients with sleep apnoea. Eur Respir J. 2006;28:159-64.

32. Stammnitz A, Jerrentrup A, Penzel T, Peter JH, Vogelmeier C, Becker HF. Automatic CPAP titration with different self-setting devices in patients with obstructive sleep apnoea. Eur Respir J. 2004;24:273-8.

33. Hertegonne KB, Rombaut B, Houtmeyers P, Van Maele G, Pevernagie DA. Titration efficacy of two auto-adjustable continuous positive airway pressure devices using different flow limitation-based algorithms. Respiration. 2008;75:48-54.

34. Fietze I, Glos M, Moebus I, Witt C, Penzel T, Baumann G. Automatic pressure titration with APAP is as effective as manual titration with CPAP in patients with obstructive sleep apnea. Respiration. 2007;74:279-86.

35. Mulgrew AT, Fox N, Ayas NT, Ryan CF. Diagnosis and initial management of obstructive sleep apnea without polysomnography: a randomized validation study. Ann Intern Med. 2007;146:157-66.

36. Gao W, Jin Y, Wang $Y$, et al. Is automatic CPAP titration as effective as manual CPAP titration in OSAHS patients? A meta-analysis. Sleep Breath. 2012;16:329-40.
37. Damiani MF, Quaranta VN, Tedeschi E, et al. Titration effectiveness of two autoadjustable continuous positive airway pressure devices driven by different algorithms in patients with obstructive sleep apnoea. Respirology. 2013;18:968-73.

38. Fox N, Hirsch-Allen AJ, Goodfellow E, et al. The impact of a telemedicine monitoring system on positive airway pressure adherence in patients with obstructive sleep apnea: a randomized controlled trial. Sleep. 2012;35:477-81.

39. Respironics. BiPAP autoSV clincal applications guide: Respironics, Inc.; 2008.

40. Teschler H, Dohring J, Wang YM, Berthon-Jones M. Adaptive pressure support servo-ventilation: a novel treatment for CheyneStokes respiration in heart failure. Am J Respir Crit Care Med. 2001;164:614-9.

41. Pepperell JC, Maskell NA, Jones DR, et al. A randomized controlled trial of adaptive ventilation for Cheyne-Stokes breathing in heart failure. Am J Respir Crit Care Med. 2003;168:1109-14.

42. Philippe C, Stoica-Herman M, Drouot X, et al. Compliance with and effectiveness of adaptive servoventilation versus continuous positive airway pressure in the treatment of Cheyne-Stokes respiration in heart failure over a six month period. Heart. 2006;92:337-42.

43. Randerath WJ, Nothofer G, Priegnitz C, et al. Long-term autoservoventilation or constant positive pressure in heart failure and coexisting central with obstructive sleep apnea. Chest. 2012;142: 440-7.

44. Sharma BK, Bakker JP, McSharry DG, Desai AS, Javaheri S, Malhotra A. Adaptive servoventilation for treatment of sleepdisordered breathing in heart failure: a systematic review and meta-analysis. Chest. 2012;142:1211-21.

45. Yoshihisa A, Suzuki S, Yamaki T, et al. Impact of adaptive servoventilation on cardiovascular function and prognosis in heart failure patients with preserved left ventricular ejection fraction and sleepdisordered breathing. Eur J Heart Fail. 2013;15:543-50.

46. Owada T, Yoshihisa A, Yamauchi H, et al. Adaptive servoventilation improves cardiorenal function and prognosis in heart failure patients with chronic kidney disease and sleepdisordered breathing. J Card Fail. 2013;19:225-32.

47. Bradley TD, Logan AG, Kimoff RJ, et al. Continuous positive airway pressure for central sleep apnea and heart failure. N Engl J Med. 2005;353:2025-33.

48. Arzt M, Floras JS, Logan AG, et al. Suppression of central sleep apnea by continuous positive airway pressure and transplant-free survival in heart failure: a post hoc analysis of the Canadian Continuous Positive Airway Pressure for Patients with Central Sleep Apnea and Heart Failure Trial (CANPAP). Circulation. 2007;115:3173-80.

49. Cowie MR, Woehrle H, Wegscheider K, et al. Rationale and design of the SERVE-HF study: treatment of sleep-disordered breathing with predominant central sleep apnoea with adaptive servoventilation in patients with chronic heart failure. Eur J Heart Fail. 2013;15:937-43.

50. http://www.resmed.com/us/en/consumer/newsandinformation/ news-releases/2015/resmed-provides-update-on-phase-iv-serve-hfstudy-of-adaptive-servo-ventilation-therapy.html. Accessed July $31,2015$.

51. http://www.fda.gov/Safety/Recalls/ucm450858.htm. Accessed July $31,2015$.

52. https://clinicaltrials.gov/ct2/show/NCT01128816. Accessed July 31, 2015.

53. Malhotra A, Bertisch S, Wellman A. Complex sleep apnea: it isn't really a disease. J Clin Sleep Med. 2008;4:406-8.

54. Javaheri S, Smith J, Chung E. The prevalence and natural history of complex sleep apnea. J Clin Sleep Med. 2009;5:205-11.

55. Allam JS, Olson EJ, Gay PC, Morgenthaler TI. Efficacy of adaptive servoventilation in treatment of complex and central sleep apnea syndromes. Chest. 2007;132:1839-46. 
56. Morgenthaler TI, Gay PC, Gordon N, Brown LK. Adaptive servoventilation versus noninvasive positive pressure ventilation for central, mixed, and complex sleep apnea syndromes. Sleep. 2007;30:468-75.

57. Brown SE, Mosko SS, Davis JA, Pierce RA, Godfrey-Pixton TV. A retrospective case series of adaptive servoventilation for complex sleep apnea. J Clin Sleep Med. 2011;7:187-95.

58. Johnson KG, Johnson DC. Bilevel positive airway pressure worsens central apneas during sleep. Chest. 2005;128:2141-50.

59. Dellweg D, Kerl J, Hoehn E, Wenzel M, Koehler D. Randomized controlled trial of noninvasive positive pressure ventilation (NPPV) versus servoventilation in patients with CPAP-induced central sleep apnea (complex sleep apnea). Sleep. 2013;36:1163-71.

60. Farney RJ, Walker JM, Boyle KM, Cloward TV, Shilling KC. Adaptive servoventilation (ASV) in patients with sleep disordered breathing associated with chronic opioid medications for nonmalignant pain. J Clin Sleep Med. 2008;4:311-9.

61. Javaheri S, Malik A, Smith J, Chung E. Adaptive pressure support servoventilation: a novel treatment for sleep apnea associated with use of opioids. J Clin Sleep Med. 2008;4:305-10.

62. Ramar K, Ramar P, Morgenthaler TI. Adaptive servoventilation in patients with central or complex sleep apnea related to chronic opioid use and congestive heart failure. J Clin Sleep Med. 2012;8: 569-76.

63. Banno K, Okamura K, Kryger MH. Adaptive servo-ventilation in patients with idiopathic Cheyne-Stokes breathing. J Clin Sleep Med. 2006;2:181-6.

64. Troitino A, Labedi N, Kufel T, El-Solh AA. Positive airway pressure therapy in patients with opioid-related central sleep apnea. Sleep Breath. 2013.
65. Carnevale C, Georges M, Rabec C, Tamisier R, Levy P, Pepin JL. Effectiveness of adaptive servo ventilation in the treatment of hypocapnic central sleep apnea of various etiologies. Sleep Med. 2011;12:952-8.

66. BiPAP autoSV-Advanced Titration guide. 2014. (Accessed 3/21/ 2014, 2014, at http://www.healthcare.philips.com/pwc_hc/us_en/ homehealth/sleep/bipapautosvadvanced/downloads/ BiPAPAutoSVAdvanced Protocol.pdf.)

67. Blau A, Minx M, Peter JG, et al. Auto bi-level pressure relief-PAP is as effective as CPAP in OSA patients - a pilot study. Sleep Breath. 2012;16:773-9.

68. Storre JH, Seuthe B, Fiechter R, et al. Average volume-assured pressure support in obesity hypoventilation: a randomized crossover trial. Chest. 2006;130:815-21.

69. Janssens JP, Metzger M, Sforza E. Impact of volume targeting on efficacy of bi-level non-invasive ventilation and sleep in obesityhypoventilation. Respir Med. 2009;103:165-72.

70. Murphy PB, Davidson C, Hind MD, et al. Volume targeted versus pressure support non-invasive ventilation in patients with super obesity and chronic respiratory failure: a randomised controlled trial. Thorax. 2012;67:727-34.

71. Ambrogio C, Lowman X, Kuo M, Malo J, Prasad AR, Parthasarathy S. Sleep and non-invasive ventilation in patients with chronic respiratory insufficiency. Intensive Care Med. 2009;35: 306-13.

72. Crisafulli E, Manni G, Kidonias M, Trianni L, Clini EM. Subjective sleep quality during average volume assured pressure support (AVAPS) ventilation in patients with hypercapnic COPD: a physiological pilot study. Lung. 2009;187:299-305.

73. Banerjee D, Yee BJ, Piper AJ, Zwillich CW, Grunstein RR. Obesity hypoventilation syndrome: hypoxemia during continuous positive airway pressure. Chest. 2007;131:1678-84. 